\title{
Effects of a school-based physical activity intervention on academic performance in 14-year old adolescents: a cluster randomized controlled trial - the School in Motion study
}

Runar Barstad Solberg ${ }^{*}$, Jostein Steene-Johannessen ${ }^{1}$, Sigmund Alfred Anderssen ${ }^{1}$, Ulf Ekelund ${ }^{1}$,

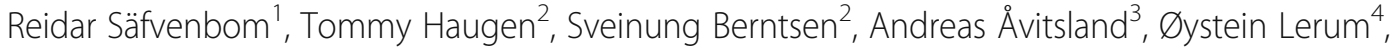
Geir Kåre Resaland ${ }^{5}$ and Elin Kolle ${ }^{1}$

\begin{abstract}
Background: School-based physical activity interventions evaluating the effect on academic performance usually includes children. We aimed to investigate the effect of a nine-month, school-based physical activity intervention titled School in Motion (SCIM) on academic performance in adolescents.

Methods: Thirty secondary schools in Norway were cluster-randomized into three groups: the Physically active learning (PAL) group ( $n=10)$, the Don't worry - Be Happy (DWBH) group $(n=10)$ or control $(n=10)$. Target dose in both intervention groups was $120 \mathrm{~min} /$ week of additional PA during school hours. Parental consent was obtained from 2084 adolescent students (76\%). Standardized national tests in reading and numeracy was conducted at baseline and at the end of the intervention. We used linear mixed model to test intervention effects. We found significant intervention effects in numeracy and reading among students in both interventions when compared with controls.
\end{abstract}

Results: The mean difference in change in numeracy was 1.7 (95\% Cl: 0.9 to 2.5; Cohen's $d=0.12$ ) and 2.0 (95\% Cl: 1.4 to 2.7; Cohen's $d=0.23$ ) points in favour of students in the PAL and DWBH intervention, respectively. Similar results were found for reading, where the mean difference in change was 0.9 ( $95 \% \mathrm{Cl} 0.2$ to 1.6; Cohen's $d=0.06$ ) and 1.1 ( $95 \% \mathrm{Cl} 0.3$ to 1.9; Cohen's $d=0.18$ ) points in favour of students in the PAL and DWBH intervention, respectively. When conducting intention to treat analysis with imputed data the estimates were attenuated and some no longer significant.

(Continued on next page)

\footnotetext{
* Correspondence: r.b.solberg@nih.no

${ }^{1}$ Department of Sports Medicine, Norwegian School of Sport Sciences, PB

4014, Ullevål Stadion, 0806 Oslo, Norway

Full list of author information is available at the end of the article
}

C C The Author(s). 2021 Open Access This article is licensed under a Creative Commons Attribution 4.0 International License, which permits use, sharing, adaptation, distribution and reproduction in any medium or format, as long as you give appropriate credit to the original author(s) and the source, provide a link to the Creative Commons licence, and indicate if changes were made. The images or other third party material in this article are included in the article's Creative Commons licence, unless indicated otherwise in a credit line to the material. If material is not included in the article's Creative Commons licence and your intended use is not permitted by statutory regulation or exceeds the permitted use, you will need to obtain permission directly from the copyright holder. To view a copy of this licence, visit http://creativecommons.org/licenses/by/4.0/. The Creative Commons Public Domain Dedication waiver (http://creativecommons.org/publicdomain/zero/1.0/) applies to the data made available in this article, unless otherwise stated in a credit line to the data. 


\begin{abstract}
(Continued from previous page)
Conclusion: The ScIM study demonstrates that two different school-based PA interventions providing approximately 120 min of additional PA weekly over nine months, significantly improved numeracy and reading performance in 14-year old students compared with controls. However, the results should be interpreted with caution as the effect sizes reported were very small or small and the estimates were attenuated when conducting intention to treat analysis. Despite this, our results are still positive and suggest that PA interventions are viable models to increase academic performance among adolescents.
\end{abstract}

Trial registration: Retrospectively registered (25/01/2019): NCT03817047.

Keywords: Physical activity, Cluster RCT, Adolescents, Academic performance

\section{Background}

Schools have received widespread attention owing to the inescapable pressure to educate students to meet accepted academic standards. Therefore, new effective teaching methods must be developed. Physical activity (PA) might be such a method because evidence has emerged concerning the positive association between PA and academic performance [1-6].

A randomized controlled trial (RCT) called Physical Activity Across the Curriculum, found improved performance in reading, numeracy, and spelling in a sub sample comparing children who received physically active lessons daily for three years with children who followed the regular curriculum [7]. Similarly, in the Fit \& Vaardig op School study, weekly physically numeracy and language lessons over two years improved numeracy and spelling performance among elementary school children [8]. These results also correspond with the Activity and Motivation in Physical Education trail findings, where the authors reported significant intervention effects on numeracy performance [9].

Despite this, the literature is ambiguous as other school-based RCTs shows no intervention effect of multicomponent PA interventions on academic performance on whole group data among children $[10,11]$ and adolescents [12]. Finally, a recent systematic review on the effects of PA interventions on academic performance in 3-16 years olds concluded that strong evidence exist of the beneficial effects of PA on numeracy performance, but the evidence is inconclusive for overall academic performance [13].

Most studies investigating the effects of school-based PA interventions on academic performance are implemented among primary school children [7, 8, 10, 11]. Studies with adolescents have focused only on PE lessons [9] or have relatively short intervention period [12]. Consequently, few large, multicomponent PA interventions that include adolescents have been implemented over longer intervention periods in lower secondary school. Therefore, we conducted a school-based cluster RCT titled School in Motion (ScIM), consisting of two multicomponent PA interventions powered to compare changes in the mean PA level among secondary schools adolescents who received two extra hours of PA per week and a control group. We recently reported a favourable effect on the daily PA level and the time spent in moderate-to-vigorous intensity physical activity (MVPA) among adolescents in one of the intervention arms compared with controls [14]. In this paper, we investigated the intervention effect of the two PA interventions on academic performance in reading and numeracy.

\section{Methods}

\section{Study design}

The ScIM study was a nine-month school-based threearm cluster RCT with schools as the cluster unit for randomization. The inclusion criteria were; $>25$ students in ninth grade. We excluded schools that worked systematically with curriculum-prescribed PA and private and special schools. When a school agreed to attend, all students in the ninth grade were invited to participate. Four collaborating partners (Norwegian School of Sport Sciences, Western Norway University of Applied Sciences, University of Agder, and University of Stavanger) conducted the study during the 2017/2018 school year.

The ScIM study was reviewed and approved by the Norwegian Centre for Research Data and adhered to the Helsinki Declaration (2008). Parents or/guardians gave written informed consent allowing their child/ward to participate. The parents or adolescents could revoke this consent at any time. ScIM is registered in ClinicalTrials. gov (25/01/2019), ID nr: NCT03817047. The design, conduct, and reporting of this trial follow the recommendations of the CONSORT statement [15]. The CONSORT checklist is in related File 1.

\section{Randomization and blinding}

Thirty schools were randomized manually by a lottery in a 1:1:1 ratio to either Physically active learning (PAL) intervention $(n=10)$, Don't worry - Be Happy (DWBH) intervention $(n=10)$ or control $(n=10)$. One school withdrew after randomization but prior to baseline testing, leaving nine schools in the control group. The 
professional who performed the randomization did not partake in other parts of the study. Neither participants, schools, the testing personnel that performed the data collection in the schools nor researchers were blinded.

\section{The ScIM interventions}

Both interventions aimed to provide approximately 120 min of additional PA in addition to the mandatory 120 to $180 \mathrm{~min}$ PE lessons per week. This goal was achieved by redistributing $5 \%$ of the other subjects to PA ( $60 \mathrm{~min}$ per week) and adding $60 \mathrm{~min}$ of PE to the curriculum. All intervention schools received $\$ 90$ per student to account for the increased expenses.

The PAL-intervention focused on increasing student PA levels and consisted of three components (Table 1). We constructed an online toolkit of activities based on student and teacher feedback, and the existing pedagogical material that teachers could use. The PAL intervention was based on a socio-ecological theoretical framework that recognizes the complex interplay between personal and environmental influences on behaviour [16]. Teachers conducting the PAL intervention were encouraged to provide activities that would be enjoyable for all students.

The focus of the DWBH-intervention was to promote friendship through PA, and it consisted of two components (Table 1): $60 \mathrm{~min}$ of a PE lesson called 'Don't worry' (DW), and $60 \mathrm{~min}$ of a lesson called 'Be happy' (BH). First, students across different classes formed groups based on their interests. The groups performed the chosen activity in the $\mathrm{BH}$ lesson throughout the intervention period. The DW lessons were similar to an ordinary PE lesson, and the activities were either the same as in the $\mathrm{BH}$ lesson or were led by students representing one of the other activity groups in the $\mathrm{BH}$ lessons. The students developed the aims, management structure, strategies for impending conflicts, and routines for registration of attendance. This intervention was anchored to an integrative relational developmental system approach to human development, promoting mutually beneficial relationships for everyone involved [17].

At least one teacher from each intervention school attended a one-day course on how to deliver the intervention. The course consisted of theoretical and practical exercises by educators with experience in integrating PA into the curriculum. Control schools continued the current practice and did not implement additional curriculum-prescribed PA.

\section{Treatment group involvement}

Teachers and students at the intervention schools were involved in the development of the two interventions in ScIM. Teachers and students were not involved designing the research questions, outcome measures, or analyses. The results of the study will be disseminated to all included schools.

\section{Delivery}

All intervention components were mandatory. Adherence to intervention components was reported in an online platform. Each week, teachers at the intervention schools self-reported components performed or not performed, and the component intensity and minutes.

\section{Measurements}

All measurements were obtained twice, first at baseline (April to August 2017) and second in the last phase of the intervention (April to June 2018). The test procedures were identical at both time points. The data were collected in the classroom and gymnasium. The research team trained all testing personnel, and all tests were conducted following the relevant guidelines.

Table 1 Intervention content and means of implementation stratified by intervention group.

\begin{tabular}{|c|c|c|c|}
\hline $\begin{array}{l}\text { Intervention components } \\
\text { (min) }\end{array}$ & $\begin{array}{l}\text { Practical } \\
\text { organization }\end{array}$ & Providers of interventions & Implementation facilitation and method \\
\hline \multicolumn{4}{|l|}{ Physically Active Learning (PAL) } \\
\hline $\begin{array}{l}\text { Physical activity in academic } \\
\text { subjects ( } 30 \text { min) }\end{array}$ & Weekly & Teachers & $\begin{array}{l}\text { An external collaborator provided a program tailored specifically } \\
\text { to the subject curriculum. } \\
\text { Teachers attended two courses during the intervention period. }\end{array}$ \\
\hline Physical education (60 min) & Weekly & Physical education teachers & Follows the normal physical education curriculum \\
\hline Physical activity (30 min) & Weekly & $\begin{array}{l}\text { Teachers/physical education } \\
\text { teachers }\end{array}$ & $\begin{array}{l}\text { Students could choose between varied activities. Teachers were } \\
\text { encouraged to motivate students during physical activity to } \\
\text { stimulate their positive feelings and attitudes towards physical } \\
\text { activity }\end{array}$ \\
\hline \multicolumn{4}{|l|}{ Don't Worry - Be Happy (DWBH) } \\
\hline $\begin{array}{l}\text { Activity class (Be happy class) } \\
\text { (60 min) }\end{array}$ & Weekly & $\begin{array}{l}\text { Teachers/physical education } \\
\text { teachers }\end{array}$ & $\begin{array}{l}\text { Self-organized activity developed according to the adolescent's } \\
\text { activity preferences. }\end{array}$ \\
\hline $\begin{array}{l}\text { Physical education (Don't } \\
\text { worry class) ( } 60 \mathrm{~min})\end{array}$ & Weekly & $\begin{array}{l}\text { Teachers/physical education } \\
\text { teachers }\end{array}$ & $\begin{array}{l}\text { Pupils led the regular PE class. Pupils practiced their Be Happy } \\
\text { activity. }\end{array}$ \\
\hline
\end{tabular}




\section{Academic performance}

Academic performance was measured using standardized computer-based national tests designed and administered by The Norwegian Directorate for Education and Training. The numeracy test measured an individual's ability to understand numbers and measurements. The reading test measured an individual's basic Norwegian reading skills, interpreting and understanding texts, and considering the form and content. Both tests included anchor questions, making it possible to provide a baseline for an equating analysis between the two timepoints. The scores were standardized to a T-score with a mean of 50 scale points with a standard deviation (SD) of 10.

\section{Anthropometry}

We measured the weight to the nearest $0.1 \mathrm{~kg}$ using a Seca 899 weight and measured the height to the nearest $0.1 \mathrm{~cm}$ using a SECA 123 Portable Stadiometer (SECA, Hamburg, Germany). We subtracted $0.6 \mathrm{~kg}$ (light clothing; gym shorts and t-shirt) or $1.5 \mathrm{~kg}$ (normal clothes; pants and sweater) from the weight measurements to account for clothing.

\section{Physical activity}

We assessed PA using ActiGraph accelerometers, models GT3X and GT3X+ (ActiGraph, LLC, Pensacola, Florida, USA). Students were instructed to wear the accelerometer on their right hip over seven consecutive days, except when sleeping, showering and bathing. ActiLife software (ActiGraph, LLC, Pensacola, Florida, USA) was used to initialize and download the accelerometer files. Raw files were processed and analysed using STATA (Stata Statistical Software, StataCorp LP), and the epoch was $10 \mathrm{~s}$. The data were recorded between 00 : 00 and 06:00, and all intervals of $\geq 20$ consecutive min with no accelerations were excluded. Days with $\geq 480$ min of active recording were considered valid. As a measure of the overall PA, we used average counts. $\mathrm{min}^{-1}(\mathrm{CPM})$ over the entire assessment period. To investigate the average minutes per day spent sedentary or in MVPA, we divided time registered with $<100$ CPM and $>1999$ CPM by the valid assessment days, respectively.

\section{Socioeconomic status}

We linked our database to the registry data collected by Statistics Norway and used the highest education level of the participants' parents as a proxy for socioeconomic status (SES). Four SES groups were computed low (primary/lower secondary/vocational high school), middle (secondary/high school), middle high (undergraduate degree), and high (graduate degree).

\section{Sample size}

The ScIM study was powered to detect changes in the primary outcome (CPM) of 7\% (49 CPM) between groups. The $\alpha$ level was 0.05 for all calculations. To detect a $49 \pm 150$ (mean \pm SD) difference in CPM between the intervention groups and control group with a power of 0.9 , expecting a dropout rate of $20 \%$, we require at least 590 participants in each intervention arm.

\section{Statistics}

In the main analysis, we included participants with a valid baseline or follow-up measures for academic performance. The data were assessed for normality and homogeneity of variance. The descriptive data are presented as the mean and SD unless otherwise stated. We fitted linear mixed models to both outcomes (numeracy and reading). Each model included fixed effects for the intervention, time (baseline and follow-up), and interaction term (intervention $\mathrm{x}$ time). As the units of randomization were schools, a "random effect" for school was included in the model, in addition to the class and subject ID to accommodate the clustering of students within these units.

We estimated the mean group values with a $95 \%$ confidence interval (CI) at the baseline and follow-up. We estimated the between group difference in change from the baseline to the follow-up between the participants in the intervention arms and control arm, adjusting for sex. Further, we examined whether sex modified the intervention effect by introducing an interaction term (timepoint $\mathrm{x}$ group $\mathrm{x}$ sex). A statistically significant interaction between sexes was evident in all academic performance models $(p<0.001$ for interaction). Consequently, we repeated the analysis stratified by this variable.

We calculated a standardized mean difference score for each specific outcome (Cohen's d), which was estimated using a random effects model. Cohen's $d$ values ranging from 0.01 to $0.20,0.20$ to 0.50 and 0.50 to 0.80 corresponds to very small, small and moderate effect size, respectively. We performed a per protocol analysis including schools with above $80 \%$ adherence to the protocol. Multiple imputations were performed on the academic performance variables as a sensitivity analysis to account for loss for the follow-up data. Imputation of variables was performed using chained equations (mi imputed chained) in Stata v.16. Mean differences in change, standard errors and 95\% CI were obtained based on 20 imputed datasets. The imputation analyses assume that data are missing at random. All statistical analyses were performed using Stata (StataCorp LP).

\section{Results}

The flow of the schools and participants is presented in Fig. 1. Among 2733 eligible students from 29 participating schools at baseline, parental consent was obtained from 


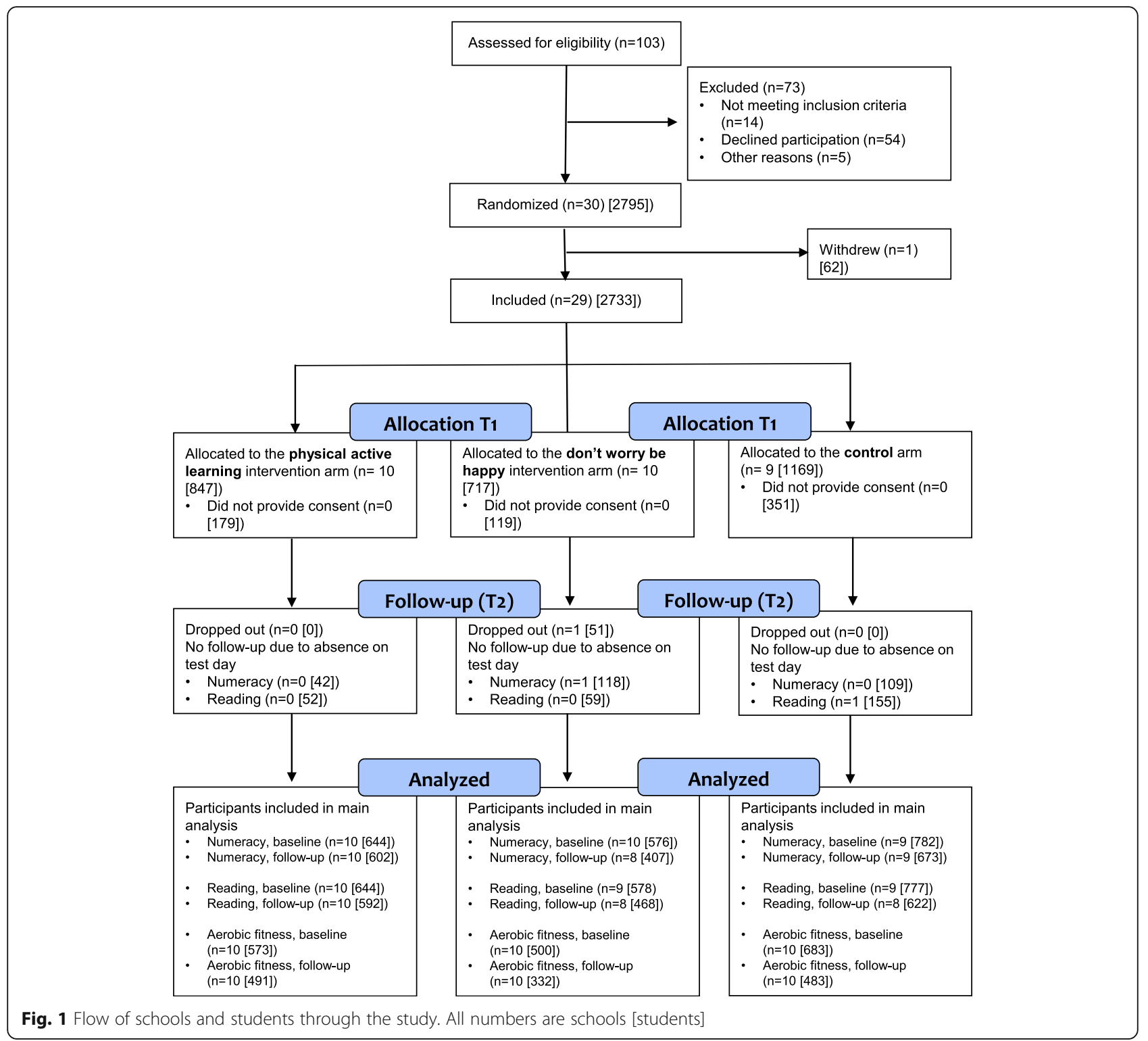

2084 students (76\%). One DWBH school withdrew from the trial after three months for practical reasons. At follow-up, one DWBH school was unable to complete the national test in numeracy and one control school were unable to complete the reading test. At baseline, 1999 and 2002 students had valid data on reading and numeracy respectively. A total of 1682 students had valid data in reading and numeracy at follow-up. The reason for the loss of follow-up data on academic performance was absences during the post-test (reading: $n=269$; numeracy $n=266$ ). The characteristics of all students by intervention group are presented in Table 2.

\section{Effects on academic performance}

We found significant intervention effects on numeracy and reading among students in both interventions compared with the control group (Fig. 2). The mean difference in change in numeracy was 1.7 (95\% CI: 0.9 to 2.5; Cohen's $\mathrm{d}=0.12$ ) and 2.0 (95\% CI: 1.4 to 2.7; Cohen's $d=0.23$ ) points in favour of students in the PAL and DWBH interventions, respectively. Similar results were found for reading, where the mean difference in change was 0.9 (95\% CI 0.2 to 1.6 ; Cohen's $d=0.06)$ and 1.1 (95\% CI: 0.3 to 1.9; Cohen's $d=0.18$ ) points in favour of students in the PAL and DWBH interventions, respectively.

Stratified by sex, the mean differences in change for numeracy in the PAL intervention were 1.0 points $(95 \%$ CI: 0.3 to 1.8 ) and 2.4 points (95\% CI: 1.5 to 3.3 ) for girls and boys respectively (Fig. 3). Similar findings were observed in the DWBH intervention, where the mean differences in change were 1.4 points (95\% CI: 0.5 to 2.2 ) 
Table 2 Participant characteristics by group allocation at baseline. Mean (SD) unless other stated.

\begin{tabular}{|c|c|c|c|c|c|c|}
\hline & \multicolumn{2}{|c|}{$\begin{array}{l}\text { PAL Intervention } \\
(n=536-655)\end{array}$} & \multicolumn{2}{|c|}{$\begin{array}{l}\text { DWBH Intervention } \\
(n=427-586)\end{array}$} & \multicolumn{2}{|c|}{$\begin{array}{l}\text { Control } \\
(n=583-795)\end{array}$} \\
\hline & Girls & Boys & Girls & Boys & Girls & Boys \\
\hline N & 328 & 327 & 286 & 300 & 387 & 408 \\
\hline Age (year) & $13.9(0.3)$ & $13.9(0.3)$ & $13.9(0.3)$ & $14.0(0.3)$ & $14.0(0.3)$ & $14.0(0.3)$ \\
\hline \multicolumn{7}{|c|}{ Parents education levels } \\
\hline Low (\%) & 5.8 & 7.0 & 5.2 & 7.6 & 2.5 & 7.1 \\
\hline Middle (\%) & 26.5 & 27.0 & 30.0 & 32.3 & 26.8 & 29.0 \\
\hline Middle high (\%) & 42.4 & 42.2 & 43.7 & 34.7 & 43.2 & 39.0 \\
\hline High (\%) & 23.8 & 22.9 & 20.2 & 24.3 & 26.1 & 23.5 \\
\hline \multicolumn{7}{|l|}{ Anthropometry } \\
\hline Height (cm) & $162.9(6.2)$ & $166.3(9.4)$ & $164.1(6.1)$ & $168.6(8.4)$ & $163.9(6.5)$ & $167.6(8.3)$ \\
\hline Weight (kg) & $54.3(9.6)$ & $54.1(11.8)$ & $55.9(10.2)$ & $56.5(11.7)$ & $54.2(9.3)$ & $54.6(11.5)$ \\
\hline \multicolumn{7}{|c|}{ Physical activity levels full day } \\
\hline Total PA (cpm) & $473.2(157.3)$ & $552.1(207.0)$ & $512.8(204.5)$ & $563.7(204.7)$ & $510.5(174.7)$ & $590.1(227.1)$ \\
\hline MVPA (min/day) & $64.5(21.8)$ & $71.6(25.8)$ & $69.6(25.0)$ & $73.3(28.0)$ & $69.7(25.5)$ & $77.8(30.3)$ \\
\hline Sedentary (min/day) & $560.0(69.9)$ & $530.3(86.7)$ & $551.3(75.4)$ & $521.7(80.6)$ & $545.9(72.0)$ & $513.9(82.9)$ \\
\hline \multicolumn{7}{|l|}{ Academic performance } \\
\hline Numeracy (points) & $53.5(9.8)$ & $56.4(9.8)$ & $54.2(9.3)$ & $55.3(9.4)$ & $56.1(9.9)$ & $55.2(10.1)$ \\
\hline Reading (points) & $56.5(9.7)$ & $54.2(9.7)$ & $55.8(9.6)$ & $53.0(10.2)$ & $57.5(9.7)$ & $52.7(10.3)$ \\
\hline
\end{tabular}

$\mathrm{PAL}=$ Physical active learning; DWBH = Don't worry - Be happy". PA = physical activity; MVPA = moderate-to-vigorous intensity physical activity

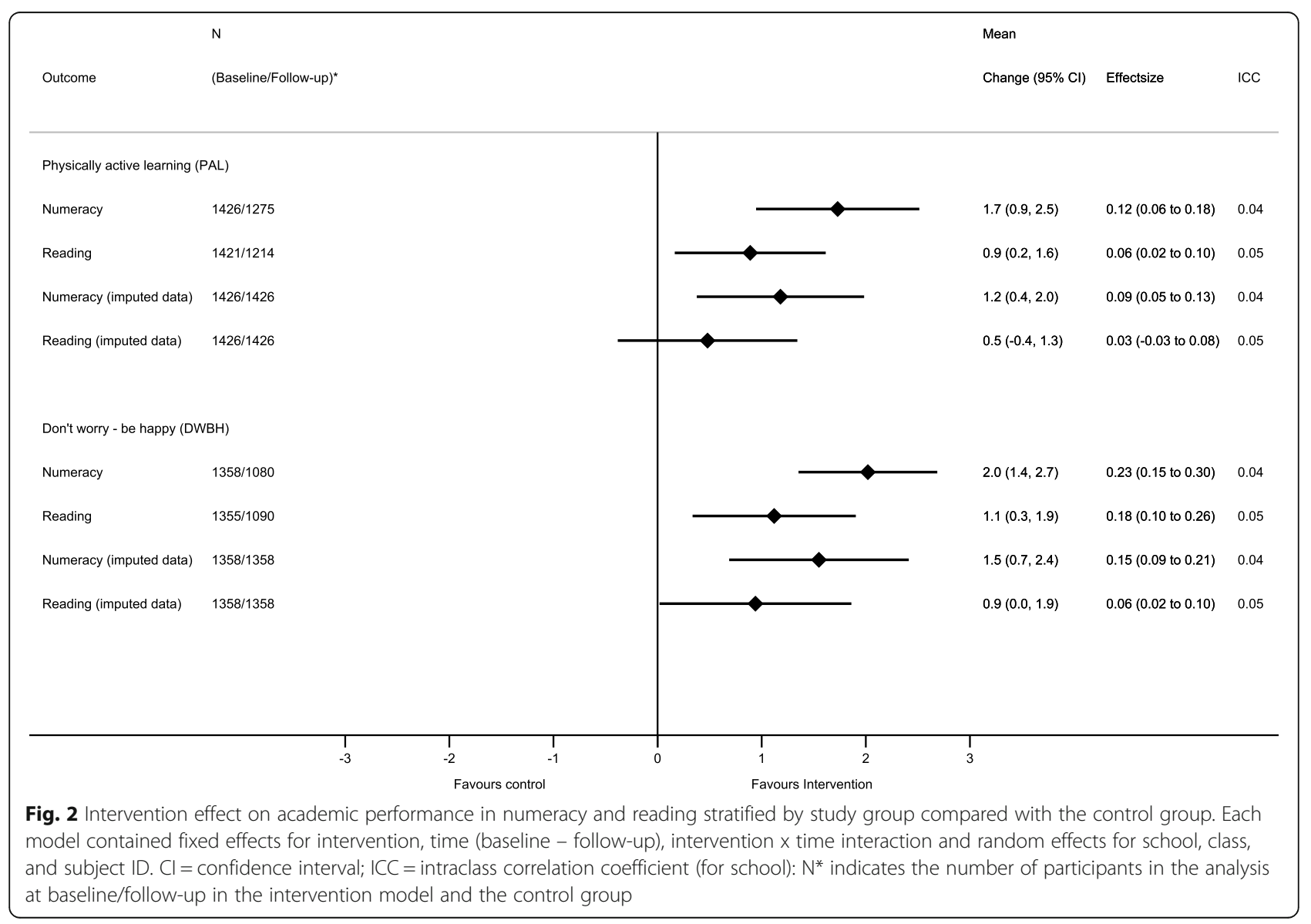




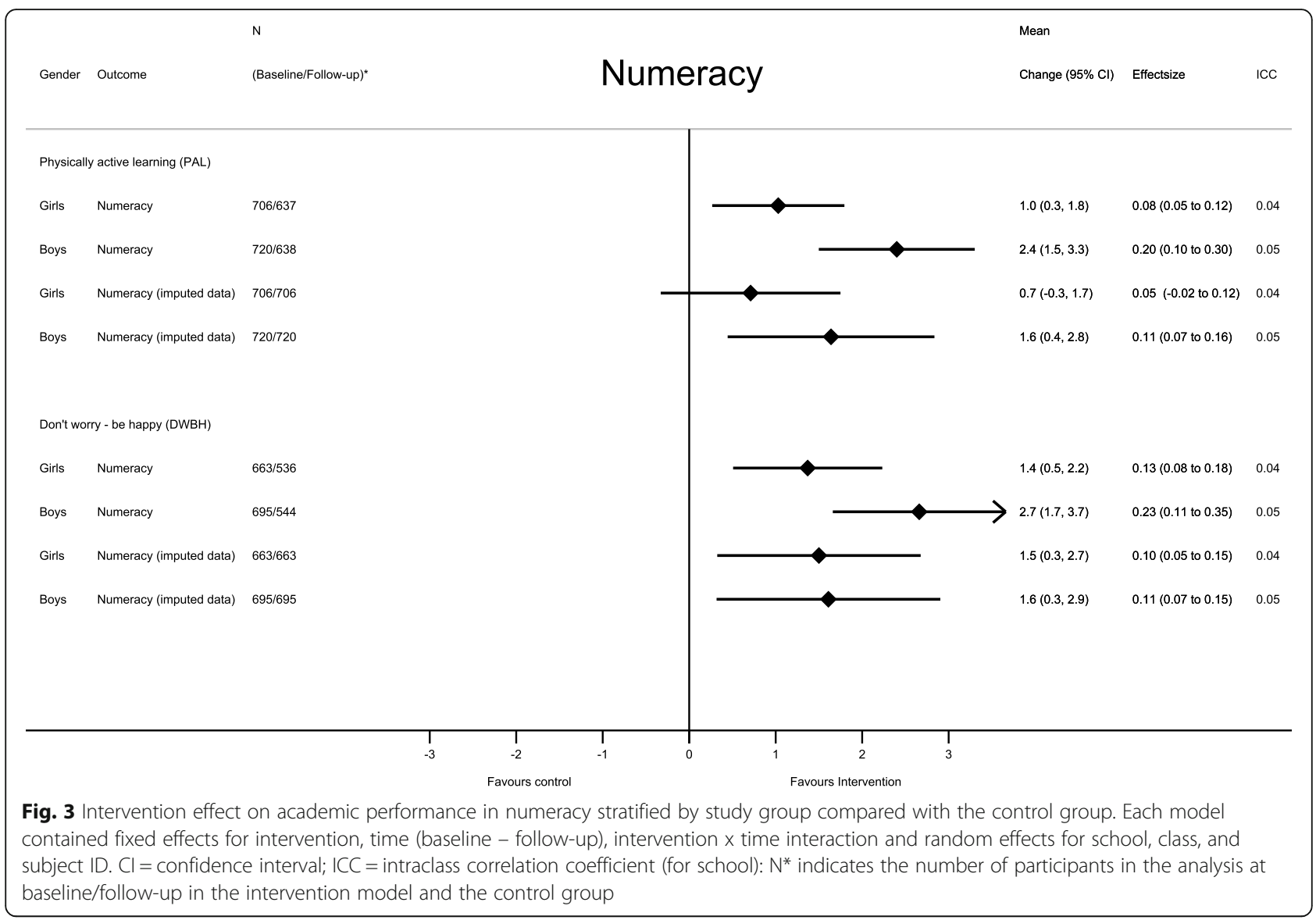

for girls and 2.7 points (95\% CI: 1.7 to 3.7 ) for boys (Fig. $3)$. The mean difference in change in reading was attenuated when compared with the overall estimates. The only significant effect when the analysis was stratified by sex was among boys in the DWBH intervention compared with control group (Fig. 4).

\section{Sensitivity and per-protocol analysis}

The sensitivity analysis from the imputed dataset followed the intention to treat (ITT) principle. Among students in the PAL-intervention, the mean difference in change in reading was attenuated to 0.5 points (95\% CI: - 0.4 to 1.3; Cohen's $d=0.03$ ) (Fig. 2) and was no longer significant in the ITT-analysis. In the sex specific analysis, we found a similar pattern for numeracy among girls in the PAL intervention and for reading among boys in the DWBH intervention, where the estimates were attenuated and no longer significant when conducting the ITT-analysis (Figs. 3 and 4). The perprotocol analysis, including schools with a delivery rate of above $80 \%$, did not differ from the main analysis (data not shown), except that the intervention did not show an intervention effect on numeracy performance among girls in the DWBH group when compared with control group (mean difference in change: $0.9,95 \% \mathrm{CI}:-0.2$ to 2.0).

\section{Intervention adherence}

The adherence to the intervention protocol was 83 and $78 \%$ for PAL and DWBH interventions, respectively. Thus, the PAL-group delivered an average of $100 \mathrm{~min} /$ week of additional PA, and the DWBH-group delivered an average of $94 \mathrm{~min} /$ week of additional PA. The adherence varied between schools, ranging from 67 to $95 \%$. Ten of the 19 intervention schools had a delivery rate of above $80 \%$.

\section{Discussion}

This paper aimed to evaluate the effect of two schoolbased PA interventions on academic performance among Norwegian adolescents. Both ScIM interventions resulted in better development over time in student academic performance in reading and numeracy than the control group.

Our findings are in line with recent intervention results suggesting a beneficial intervention effect of school-based PA on academic performance [7-9]. Although our results align with some studies, other studies do not support our findings [10-12]. The LCoMotion 


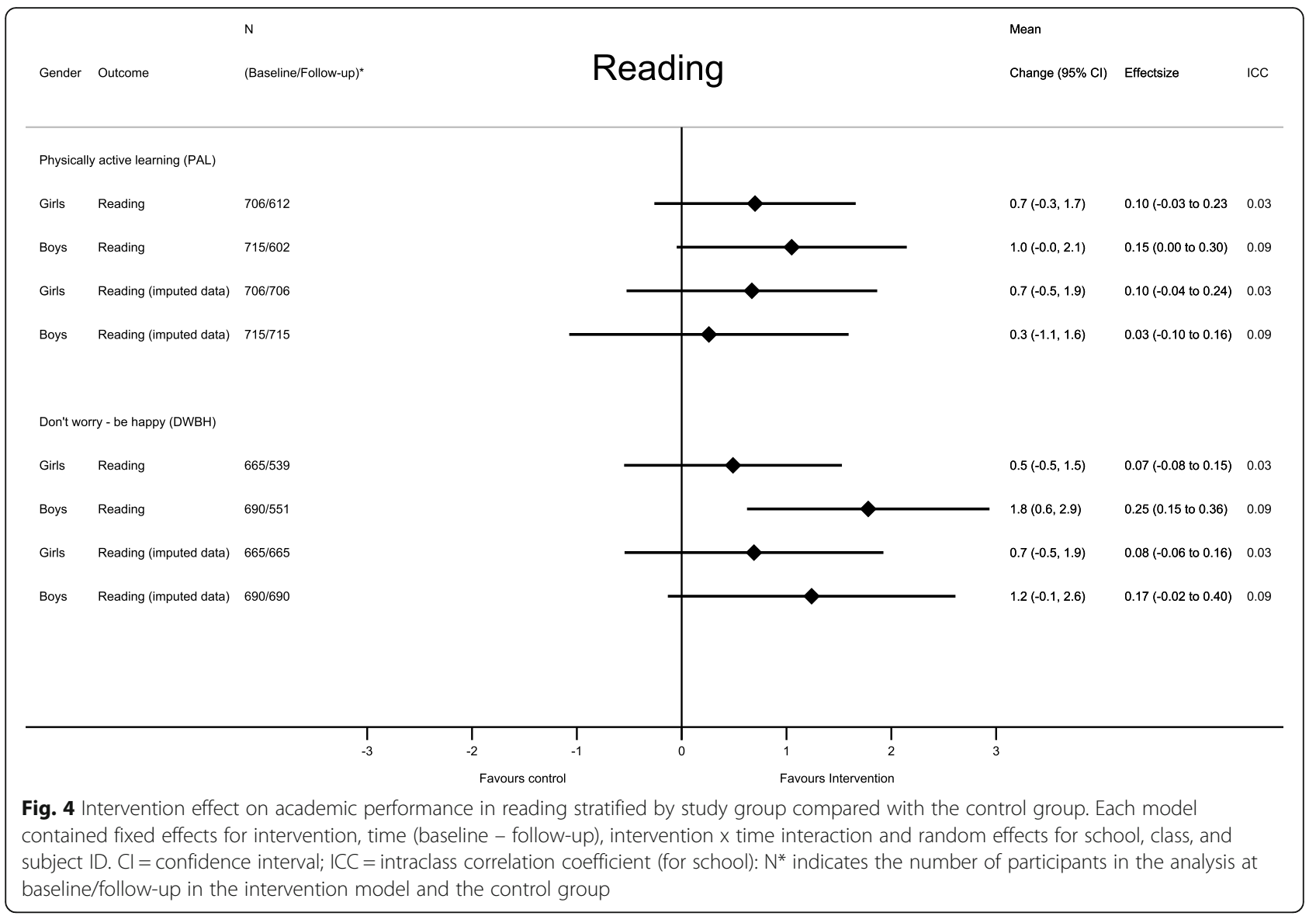

study included 632 Danish adolescents who performed 60 min of additional PA each school day over 20 weeks [12], The Active Smarter Kids study included 1100 Norwegian fifth graders who carried out $165 \mathrm{~min}$ of additional PA per week over seven months [11] and the Academic Achievement and Physical Activity Across the Curriculum study included 584 American children who engaged in more than $100 \mathrm{~min}$ of PA each week over three years [10]. The discrepancy in intervention effects could be due to several factors.

First, studies with non-significant results on academic performance consisted of various types of PA components, physically active learning, PA after school, active transportation and homework, short PA breaks during theoretical lessons, and recess [10-12]. Although no clear evidence indicate that some components of schoolbased PA would be more effective than others, AlvarezBueno et al. [18] concluded that all PA components, but especially $\mathrm{PE}$, could improve academic performance. In ScIM, both interventions included additional PE, which LCoMotion, The Active Smarter Kids and Academic Achievement and Physical Activity Across the Curriculum study did not. However, neither did Physical Activity Across the Curriculum study [7] or the Fit \& Vardig op School intervention [8], making it difficult to conclude which components of school-based PA are most effective.

Second, adherence to protocol might be of importance. In the LCoMotion [12], Academic Achievement and Physical Activity Across the Curriculum [10], and The Active Smarter Kids [11] studies, the teachers delivered an average of 40,55 , and $80 \%$, respectively, of the weekly target dose across the intervention period. This adherence to protocol is lower than that reported in ScIM. When considering the per-protocol analyses it is important to emphasize that adherence was selfreported by the teachers every week across the intervention. Consequently, the self-report could be subject to bias; however, it is the same method used in the comparable studies. When designing and planning the ScIM interventions, we conducted a five-month pilot study, including seven schools and 700 adolescents, which led to adjusting both models to better reach the target group. The intervention models were simplified (i.e. one component - physically active breaks - was removed from the PAL model), and more resources were given to the teachers to increase adherence to the protocol. In ScIM, the teachers delivered approximately $80 \%$ of the intervention dose. The main reason for not reaching the intended target dose was various special events 
throughout the year (i.e., exams, holidays, and school trips). Nevertheless, ScIM indicates that it is possible to implement school-based PA interventions that positively affect academic performance in an already busy curriculum.

Third, the PAL intervention resulted in better daily PA development over time than the control group [14]. This results was also observed in the Physical Activity Across the Curriculum study [7], but was not reported in the other studies [11, 12]. The increased PA levels in the PAL model could theoretically be linked to changes in the brain structure, function or neurotransmitters concentration that occurs in students who are more physically active [19]. Furthermore, PA can affect the brain's physiology by increasing the cerebral capillary growth, blood flow and nerve cells in the hippocampus, supporting learning and memory related to academic performance $[19,20]$.

In the DWBH intervention, we found effects on academic performance despite no effect on PA levels. However, the focus in the DWBH-intervention was to promote friendships through $\mathrm{PA}$, which was more important than the dose and intensity of the activities. We speculate that an alternative explanation for the intervention effect on academic performance in the DWBH intervention is that the self-elected activities may have enhanced arousal, minimized fatigue and boredom, and led to higher levels of self-efficacy, which could optimize student academic performance [21]. Furthermore, the chosen activities may have encouraged students to cooperate with classmates, employ strategies and adapt to changing task demands. Studies have reported that PE enriched with social interaction improves inhibition [22, 23]. In addition, PA is associated with planning performance [24] and cognitive flexibility [25], which relates to better academic performance. Another possible mechanism is that varied PA through the curriculum enhances the enjoyment of academic lessons, leading to higher motivation and engagement with theoretical materials. This outcome can improve the classroom climate and subsequently act as a confounder for the intervention effect. However, when we rerun the analysis with adjustments for the classroom climate, the results did not change from the main analysis.

Our results suggest that the PA content and relational quality seems to be as important as the dose and intensity when aiming to increase students' academic performance. These findings indicate that it is possible to develop new active teaching methods which could be more effective for increasing academic performance compared to more traditionally sedentary teaching methods. However, given limitations in small effect sizes and relative short intervention duration, more research is warranted. Studies implemented over a longer time period (e.g. two or three years) with direct measurement of cognition and other possible mechanisms can provide a more in depth understanding of how PA can affect academic performance among adolescents and should be prioritized.

The strengths of this study include the cluster randomized design using two different PA interventions, the high adherence to the protocols, and device measured PA, which ensures internal and external validity. Additionally, the large sample size $(76 \%$ of eligible students) consisting of an understudied population recruited from four different regions across Norway reduces the risk of bias and suggests that the findings are generalizable to some extent. Finally, to provide an unbiased estimate of group allocation, we performed a mixed model analysis with all participants with valid data on either time point for academic performance. Multiple imputation when performing mixed models analyses can lead to unstable results [26], and when the analysis was rerun on the imputed data the estimates were attenuated and some no longer significant.

We did not include any measurement of cognition or biological pathways on how PA might influence academic performance. Furthermore, although several studies have used national tests to measure academic performance, these could be a potential limitation because no validation studies exist. Another limitation is that the effect size (Cohen's d) of the intervention effect in the primary analysis is considered very small or small $(\mathrm{d}=0.06$ to 0.23 ). However, other intervention studies that have demonstrated beneficial effects on academic performance have been implemented over two school years. As our intervention only lasted for nine months, we can speculate regarding whether a longer intervention duration would result in further improvements in academic performance.

\section{Conclusion}

The ScIM study demonstrates that two different schoolbased PA interventions providing approximately $120 \mathrm{~min}$ of additional PA weekly over nine months, significantly improved numeracy and reading performance in 14-year old students compared with controls. However, the results should be interpreted with caution as the effect sizes reported were very small or small and the estimates were attenuated when conducting ITT analysis. Despite this, our results are still positive and suggest that PA interventions are viable models to increase academic performance among adolescents.

\section{Abbreviations}

DWBH: Don't worry - be happy; MVPA: Moderate-to-vigorous intensity physical activity; PA: Physical activity; PAL: Physically active learning; PE: Physical education; RCT: Randomized controlled trial; ScIM: School in motion 


\section{Acknowledgements}

We are grateful to all the adolescents and teachers who participated in this study for their time and effort allowing us to complete this study. A special thanks goes to the teachers at the intervention schools for the great job they did in delivering the PA intervention. We would also like to thank all students and research assistants who participated in the data collection. We are also grateful to the ScIM Study group. The study was funded by the Norwegian Directorate for Education and Training.

\section{Authors' contributions}

Each author has contributed to the conception and design of the work. RS conducted the analysis and wrote the first draft of the paper and contributed revisions to the manuscript. JSJ, SAA, UE, RS, TH, SV, AA, ØL, GKR, EK participated in writing of the paper and approved the final version.

\section{Funding}

The study was funded by the Norwegian Directorate for Education and Training. The funding body had no involvement in design of the study, data collection, analysis, interpretation of data or in the drafting of the manuscript.

\section{Availability of data and materials}

The datasets generated and/or analyzed during the current study are not publicly available as publications are planned but are available from the corresponding author on reasonable request.

\section{Declarations}

\section{Ethics approval and consent to participate}

The ScIM study was reviewed and approved by the Norwegian Centre for Research Data and adhered to the Helsinki Declaration (2008). The trial's protocol was retrospectively registered at ClinicalTrials.gov on 01/25/2019 (NCT03817047). Written informed consent from the participants and their parents or caretakers was obtained prior to the data collection.

\section{Consent for publication}

Not applicable.

\section{Competing interests}

The authors declare that they have no competing interests.

\section{Author details}

'Department of Sports Medicine, Norwegian School of Sport Sciences, PB 4014, Ullevål Stadion, 0806 Oslo, Norway. ${ }^{2}$ Faculty of Health and Sport Science, Department of Sport Science and Physical Education, University of Agder, PB 422, 4604 Kristiansand, Norway. ${ }^{3}$ Department of Education and Sport Science, University of Stavanger, Pb 8600 Forus, 4036 Stavanger, Norway. ${ }^{4}$ Department of Sport, Food and Natural Sciences, Faculty of Education, Arts and Sports, Western Norway University of Applied Sciences, Campus Sogndal, Box 133, 6851 Sogndal, Norway. ${ }^{5}$ Center for Physically Active Learning, Faculty of Education, Arts and Sports, Western Norway University of Applied Sciences, Campus Sogndal, 6856 Sogndal, Norway.

\section{Received: 14 December 2020 Accepted: 19 April 2021}

\section{Published online: 06 May 2021}

\section{References}

1. Booth JN, Leary SD, Joinson C, Ness AR, Tomporowski PD, Boyle JM, et al. Associations between objectively measured physical activity and academic attainment in adolescents from a UK cohort. Br J Sports Med. 2014;48(3): 265-70. https://doi.org/10.1136/bjsports-2013-092334.

2. Maher C, Lewis L, Katzmarzyk PT, Dumuid D, Cassidy L, Olds T. The associations between physical activity, sedentary behaviour and academic performance. J Sci Med Sport. 2016;19(12):1004-9. https://doi.org/10.1016/j. jsams.2016.02.010.

3. Kwak $L$, et al. Associations between physical activity, fitness, and academic achievement. J Pediatr. 2009;155(6):914-918.e1.

4. Haapala EA, et al. Longitudinal associations of physical activity and pubertal development with academic achievement in adolescents. J Sport Health Sci. 2019;9(3):265-73.
5. Norris E, et al. Physically active lessons in schools and their impact on physical activity, educational, health and cognition outcomes: a systematic review and meta-analysis. Br J Sports Med. 2020;54(14):826-38.

6. Sneck S, Viholainen H, Syväoja H, Kankaapää A, Hakonen H, Poikkeus AM, et al. Effects of school-based physical activity on mathematics performance in children: a systematic review. Int J Behav Nutr Phys Act. 2019;16(1):109. https://doi.org/10.1186/s12966-019-0866-6.

7. Donnelly JE, Greene JL, Gibson CA, Smith BK, Washburn RA, Sullivan DK, et al. Physical activity across the curriculum (PAAC): a randomized controlled trial to promote physical activity and diminish overweight and obesity in elementary school children. Prev Med. 2009;49(4):336-41. https://doi.org/10.1016/j.ypmed.2009.07.022.

8. Mullender-Wijnsma MJ, et al. Physically active math and language lessons improve academic achievement: a cluster randomized controlled trial. Pediatrics. 2016;137(3):e20152743.

9. Lubans DR, et al. School physical activity intervention effect on adolescents' performance in mathematics. Med Sci Sports Exerc. 2018; 50(12):2442-50

10. Donnelly JE, Hillman CH, Greene JL, Hansen DM, Gibson CA, Sullivan DK, et al. Physical activity and academic achievement across the curriculum: results from a 3-year cluster-randomized trial. Prev Med. 2017;99:140-5. https://doi.org/10.1016/j.ypmed.2017.02.006.

11. Resaland GK, Aadland E, Moe VF, Aadland KN, Skrede T, Stavnsbo M, et al. Effects of physical activity on schoolchildren's academic performance: the active smarter kids (ASK) cluster-randomized controlled trial. Prev Med. 2016; 91:322-8. https://doi.org/10.1016/j.ypmed.2016.09.005.

12. Tarp J, Domazet SL, Froberg K, Hillman CH, Andersen LB, Bugge A. Effectiveness of a school-based physical activity intervention on cognitive performance in Danish adolescents: LCoMotion-learning, cognition and motion - a cluster randomized controlled trial. PLoS One. 2016;11(6): e0158087. https://doi.org/10.1371/journal.pone.0158087.

13. Singh AS, Saliasi E, van den Berg V, Uijtdewilligen $L$, de Groot RHM, Jolles $J$, et al. Effects of physical activity interventions on cognitive and academic performance in children and adolescents: a novel combination of a systematic review and recommendations from an expert panel. Br J Sports Med. 2019:53(10):640-7. https://doi.org/10.113 6/bjsports-2017-098136.

14. Kolle $E$, et al. The effect of a school-based intervention on physical activity, cardiorespiratory fitness and muscle strength: the School in Motion cluster randomized trial. Int J Behav Nutr Phys Act. 2020;17(1):154.

15. Campbell MK, Piaggio G, Elbourne DR, Altman DG, for the CONSORT Group. Consort 2010 statement: extension to cluster randomised trials. BMJ. 2012; 345:e5661. https://doi.org/10.1136/bmj.e5661.

16. McLeroy KR, Bibeau D, Steckler A, Glanz K. An ecological perspective on health promotion programs. Health Educ Q. 1988;15(4):351-77. https://doi. org/10.1177/109019818801500401.

17. Overton, W., Processes, Relations, and Relational-Developmental-Systems. 2015. p. 9-62.

18. Alvarez-Bueno C, et al. Academic achievement and physical activity: a metaanalysis. Pediatrics. 2017;140(6):e20171498.

19. Chaddock L, Pontifex MB, Hillman CH, Kramer AF. A review of the relation of aerobic fitness and physical activity to brain structure and function in children. J Int Neuropsychol Soc. 2011;17(6):975-85. https://doi.org/10.1017/ S1355617711000567.

20. Hillman $\mathrm{CH}$, Biggan JR. A review of childhood physical activity, brain, and cognition: perspectives on the future. Pediatr Exerc Sci. 2017;29(2):170-6. https://doi.org/10.1123/pes.2016-0125.

21. Fedewa AL, Ahn S. The effects of physical activity and physical fitness on children's achievement and cognitive outcomes: a meta-analysis. Res Q Exerc Sport. 2011;82(3):521-35. https://doi.org/10.1080/02701367.2011.1 0599785.

22. Crova C, Struzzolino I, Marchetti R, Masci I, Vannozzi G, Forte R, et al. Cognitively challenging physical activity benefits executive function in overweight children. J Sports Sci. 2014;32(3):201-11. https://doi.org/10.1080/ 02640414.2013.828849.

23. van der Niet AG, Smith J, Oosterlaan J, Scherder EJA, Hartman E, Visscher C. Effects of a cognitively demanding aerobic intervention during recess on Children's physical fitness and executive functioning. Pediatr Exerc Sci. 2016; 28(1):64-70. https://doi.org/10.1123/pes.2015-0084.

24. van der Niet AG, Smith J, Scherder EJA, Oosterlaan J, Hartman E, Visscher C. Associations between daily physical activity and executive functioning in 
primary school-aged children. J Sci Med Sport. 2015;18(6):673-7. https://doi. org/10.1016/j.jsams.2014.09.006.

25. Schmidt M, Jäger K, Egger F, Roebers CM, Conzelmann A. Cognitively engaging chronic physical activity, but not aerobic exercise, affects executive functions in primary school children: a group-randomized controlled trial. J Sport Exerc Psychol. 2015;37(6):575-91. https://doi.org/1 0.1123/jsep.2015-0069

26. Twisk J, de Boer M, de Vente W, Heymans M. Multiple imputation of missing values was not necessary before performing a longitudinal mixedmodel analysis. J Clin Epidemiol. 2013;66(9):1022-8. https://doi.org/10.1016/j. jclinepi.2013.03.017.

\section{Publisher's Note}

Springer Nature remains neutral with regard to jurisdictional claims in published maps and institutional affiliations.

Ready to submit your research? Choose BMC and benefit from:

- fast, convenient online submission

- thorough peer review by experienced researchers in your field

- rapid publication on acceptance

- support for research data, including large and complex data types

- gold Open Access which fosters wider collaboration and increased citations

- maximum visibility for your research: over $100 \mathrm{M}$ website views per year

At BMC, research is always in progress.

Learn more biomedcentral.com/submissions 